\title{
Efecto de un complejo multienzimático y un probiótico en gallinas de postura alimentadas con dietas sorgo-soya-canola
}

\author{
Pedro Juárez Morales ${ }^{a}$ \\ Arturo Cortes Cuevas ${ }^{a^{*}}$ \\ José Arce Menocal ${ }^{b}$ \\ Juan Carlos Del Río García ${ }^{c}$ \\ Gabriela Gómez Verduzco ${ }^{\mathrm{a}}$ \\ Ernesto Avila González ${ }^{\text {a }}$
}

a Universidad Nacional Autónoma de México, Facultad de Medicina Veterinaria y Zootecnia, Centro de Enseñanza, Investigación y Extensión en Producción Avícola. Manuel M. López s/n Col. Zapotitlán, 13209, Tláhuac, Ciudad de México, México.

${ }^{\mathrm{b}}$ Universidad Michoacana de San Nicolás de Hidalgo, Facultad de Medicina Veterinaria y Zootecnia. Morelia, Michoacán, México.

${ }^{\mathrm{c}}$ Universidad Nacional Autónoma de México, Facultad de Estudios Superiores Cuautitlán. Estado de México, México.

*Autor de correspondencia: cortesca68@gmail.com

\section{Resumen:}

Se realizó un estudio con la finalidad de evaluar los parámetros productivos, concentraciones de IgA secretora intestinal, colesterol, LDL, HDL en suero, de gallinas de postura alimentadas con dietas sorgo + soya + canola con menor contenido de nutrientes adicionadas con un complejo multienzimático (proteasas, amilasas y xilanasas) y un probiótico (Bacillus subtilis). Se emplearon 180 gallinas Bovans White de 42 a 54 semanas de edad distribuidas en un diseño completamente al azar, con 3 tratamientos: 1) dieta testigo, 2) dieta reducida en energía metabolizable $(50 \mathrm{kcal} / \mathrm{kg}$ y $2 \%$ de proteína y 
aminoácidos metionina y lisina) + enzimas, 3 ) dieta 2 + probiótico. Para los resultados de rendimiento productivo existió diferencia $(P<0.05)$ en el peso de huevo con menor peso el tratamiento 2, pero con la adición de enzimas y probióticos, se incrementó. Para las variables inmunidad humoral, colesterol, LDL y HDL, no se observó diferencia estadística $(P>0.05)$ entre tratamientos. Se puede concluir que la inclusión de enzimas y probiótico permitió reducir nutrientes (EM, proteína y aminoácidos lisina y metionina) con resultados similares en parámetros productivos respecto a la dieta testigo, y sin cambios en inmunidad intestinal, niveles de colesterol, lipoproteínas de alta y baja densidad en gallinas Bovans White.

Palabras clave: Enzimas, Bacillus subtilis, Gallina de postura, Inmunidad, Colesterol.

Recibido: 05/04/2018

Aceptado: 26/06/2019

\section{Introducción}

El empleo de enzimas exógenas en dietas para aves, se ha incrementado en la última década con el fin de incrementar la digestibilidad de la energía y la proteína ${ }^{(1,2)}$. Los polisacáridos no amiláceos (PNA) son los componentes mayores de la fibra en los ingredientes tradicionales; estudios previos indican que el sorgo contiene alrededor de $6.5 \%$ y la pasta de soya $3.3 \%$ de estos polisacáridos ${ }^{(3)}$. Los granos de cereales consisten en arabinoxilanos y glucanos; para la soya y canola en arabinos, arabinogalactanos, galactanos, mananos y pectinas $^{(4,5)}$. Los PNA de las paredes celulares, tales como los arabinoxilananos solubles e insolubles se degradan por las xilanasas, liberando nutrientes encapsulados dentro de la pared celular y con mejora en el acceso a las enzimas endógenas ${ }^{(6,7)}$. Las enzimas son ampliamente utilizadas para degradar los PNA en la industria de los alimentos, son xilanasas y $\beta$-glucanasas ${ }^{(8)}$ y reducen la pérdida de aminoácidos endógenos ${ }^{(9)}$.

Los productos con actividad multienzimática, han demostrado que la combinación de xilanasas, amilasas y proteasas mejoran la digestibilidad en dietas para aves ${ }^{(10,11,12)}$.

Amerah $e t a l^{(9)}$ informaron que el empleo de proteasas, amilasas y xilanasas en dietas maízpasta de soya mejoraron la digestibilidad ileal de proteína, energía y retención de nitrógeno en pollos de engorda, lo que se tradujo en un mayor rendimiento productivo.

La inclusión en la dieta de las aves con bacterias benéficas como las del género Bacillus, es una alternativa al empleo de antibióticos promotores del crecimiento ${ }^{(13,14,15)}$. 
Estudios recientes de un producto comercial basado en tres cepas de Bacillus, ha mostrado efectos positivos en dietas maíz-soya ${ }^{(16)}$. Dado que enzimas exógenas y probióticos se incluyen en la dietas comerciales para aves, hay poca información de la interacción entre enzimas exógenas y probióticos; a las enzimas se les atribuye un efecto probiótico en el intestino del pollo ${ }^{(12,17)}$. Además modulan la respuesta inmune y suprimen las reacciones inmunes inflamatorias en las paredes intestinales ${ }^{(18)}$.

Por otra parte, se ha informado que la adición de probióticos en gallinas ponedoras, mejoran la conversión alimenticia, calidad del huevo (disminución del nivel de colesterol en yema, incremento en el grosor de cascarón y peso del huevo), y disminución de las concentraciones de colesterol en sangre ${ }^{(19)}$.

El objeto de este estudio fue estudiar, el efecto de la inclusión solo o en combinación de un complejo multienzimático (xilanasas, proteasas y amilasas) y un probiótico (Bacillus subtilis) en dietas sorgo-soya-canola con menor contenido de energía y proteína para gallinas Bovans White, sobre los parámetros productivos, producción de IgA secretora intestinal y contenido de colesterol, LDL, HDL en suero.

\section{Material y métodos}

El experimento se realizó en las instalaciones del Centro de Enseñanza, Investigación y Extensión en Producción Avícola (CEIEPAv) de la Facultad de Medicina Veterinaria y Zootecnia de la Universidad Nacional Autónoma de México (UNAM). Se utilizaron 180 gallinas Bovans White de 42 semanas de edad, las cuales se distribuyeron en un diseño completamente al azar con tres tratamientos con 5 réplicas de 12 gallinas cada una, los cuales se distribuyeron de la siguiente manera: 1) dieta testigo, 2) dieta reducida en energía metabolizable $(50 \mathrm{kcal} / \mathrm{kg})$, así como en proteína y aminoácidos limitantes metionina y lisina ( $2 \%$ de lo empleado en la dieta testigo) + enzimas y 3$)$ dieta $2+$ enzimas + probiótico.

Las dietas utilizadas muestran en el Cuadro 1. La dieta testigo positivo cumpliendo las recomendaciones de la estirpe Bovans White para la etapa productiva y otra dieta testigo reducida en energía metabolizable (EM), proteína y aminoácidos esenciales. Se nota que la reducción de dichos nutrientes disminuyó considerablemente la cantidad de pasta de soya y aceite vegetal en la dieta. Ambas dietas fueron tipo comercial e incluyeron fitasa.

Se adicionaron dos productos comerciales a las dietas reducidas en nutrientes; un complejo enzimático (Axtra XAP $101 \mathrm{TPT}$, Dupont, Animal Nutrition), a razón de $250 \mathrm{~g} / \mathrm{t}$ de alimento, que contiene xilanasas (20,000 u/g derivados de Trichoderma longibrachiatum), proteasas $(40,000 \mathrm{u} / \mathrm{g}$ derivados de Bacillus subtilis) y amilasas $(2,000 \mathrm{u} / \mathrm{g}$ derivados de 
Bacillus licheniformis) y un probiótico que contiene tres cepas de B. subtilis (Enviva PRO $201 \mathrm{GT}^{\mathrm{R}}$, Dupont, Animal Nutrition 3E+08 ufc/g) a razón de $250 \mathrm{~g} / \mathrm{t}$ de alimento.

Cuadro 1: Composición y análisis calculados de dietas basales sorgo-pasta de soya-pasta de canola, en gallinas de postura de 42 a 54 semanas de edad, adicionadas con un complejo multienzimático y un probiótico

\begin{tabular}{lll}
\hline Ingredientes & Testigo $+(\mathrm{kg})$ & Testigo $-(\mathrm{kg})$ \\
\hline Sorgo & 653.780 & 675.850 \\
Pasta de soya & 148.520 & 136.950 \\
Canola & 58.160 & 58.160 \\
Aceite vegetal & 13.720 & 3.000 \\
Ortofosfato de calcio & 9.190 & 9.200 \\
Carbonato de calcio & 105.530 & 105.560 \\
Sal & 4.320 & 4.320 \\
DL-Metionina 99\% & 1.340 & 1.310 \\
L-lisina HCl 78\% & 0.470 & 0.670 \\
Vitaminas y minerales $*$ & 2.400 & 2.400 \\
Bacitracina MD 10\% & 0.500 & 0.500 \\
Cloruro de colina 60\% & 0.500 & 0.500 \\
Pigmento rojo chile $* *$ & 0.800 & 0.800 \\
Larvicida & 0.500 & 0.500 \\
Antioxidante BHT & 0.150 & 0.150 \\
Apo-ester 10\% & 0.050 & 0.050 \\
Fitasa & 0.045 & 0.045 \\
\hline Total & 1000 & 1000 \\
\hline & & Análisis calculado \\
\hline Energía metabolizable, kcal/kg & 2800 & 2750 \\
Proteína cruda, \% & 15.79 & $15.41 * * *$ \\
Lisina total, \% & 0.73 & $0.71 * * *$ \\
Met+Cist total, \% & 0.67 & $0.66 * * *$ \\
Calcio total, \% & 4.25 & 4.25 \\
Fósforo disponible, $\%$ & 0.46 & 0.46 \\
Sodio, \% & 0.18 & 0.18 \\
\hline & & \\
\hline
\end{tabular}

* Vit A, 3000000 UI; Vit D3,750 000 UI; Vit E, 6000 UI; Vit K3, 1.0 g; niacina, 25 g; biotina, 0.063 g; cloruro de colina, $250 \mathrm{~g}$; selenio, $0.2 \mathrm{~g}$; cobalto, $0.1 \mathrm{~g}$; yodo, $0.3 \mathrm{~g}$; cobre, $10 \mathrm{~g}$; zinc, $50 \mathrm{~g}$; hierro, $100 \mathrm{~g}$; manganeso, $100 \mathrm{~g}$; vehículo c.b.p 1,000.00 g.

**Pigmento rojo vegetal (avired en polvo) colorante de origen vegetal $5 \mathrm{~g} / \mathrm{kg}$, Capsicum.

***Reducción del $2 \%$ respecto a lo contenido en la dieta testigo. 
Las aves se alojaron en una caseta de ambiente natural, en jaulas de tres gallinas cada una, por un periodo de 12 semanas. Se les proporcionó luz artificial y luz natural para un total de $16 \mathrm{~h}$ diarias. Se les alimentó con las dietas experimentales sorgo-soya-canola en forma de harina, a razón de $105 \mathrm{~g} / \mathrm{ave} /$ día. La administración de agua fue a libertad.

Semanalmente, durante las 12 semanas del estudio, se registraron y resumieron los datos de producción; peso del huevo, consumo de alimento por ave/día e índice de conversión. Al final del estudio, se utilizaron 20 huevos por tratamiento, a los cuales se les determinó el grosor de cascarón sin las membranas internas con un micrómetro manual, las unidades Haugh y color de yema; utilizando el sistema automatizado QCM+ de la empresa Technical Services y Supplies Inc (TSS).

Para evaluar la respuesta de anticuerpos en intestino, al final de la prueba, se seleccionaron cinco gallinas por tratamiento y se procesaron en el rastro de acuerdo a lo establecido en la Norma Oficial Mexicana, NOM-033-ZOO-1995 Sacrificio humanitario de los Animales Domésticos y Silvestres. Se obtuvieron $10 \mathrm{~cm}$ de íleon, para posteriormente realizar lavados con $10 \mathrm{ml}$ de solución salina isotónica SSI fría y estéril, pasando tres veces la SSI a través del lumen intestinal, la solución se recolectó y se congeló a $-20^{\circ} \mathrm{C}$, hasta su posterior evaluación con la prueba de ELISA siguiendo el procedimiento descrito previamente por Gómez ${ }^{(20)}$.

A las 54 semanas de edad, se tomaron también muestras de sangre de 30 gallinas (10 gallinas por tratamiento), cada muestra se centrifugó a 3,000 rpm/10 min, posteriormente se obtuvo el suero, para determinar los niveles de colesterol sérico, LDL y HDL, en el Departamento de Patología de la Facultad de Medicina Veterinaria y Zootecnia UNAM. Los resultados obtenidos se transformaron de $\mathrm{mmol} / \mathrm{dl} \mathrm{a} \mathrm{mg} / \mathrm{dl}$ mediante el factor de conversión 0.0259 .

En variables de producción, colesterol en yema de huevo, colesterol sérico, LDL y HDL en suero, se analizaron mediante un diseño completamente al azar, por medio del paquete estadístico IBM SPSS Statistics v. 19 $9^{(21)}$, utilizando el análisis de varianza conforme a un diseño completamente al azar y las diferencias entre tratamientos, se compararon por medio de la prueba de Tukey. 


\section{Resultados}

En el Cuadro 2, se muestran los datos obtenidos en 84 días de experimentación. Se puede apreciar que los resultados obtenidos para porcentaje de postura, consumo de alimento y conversión alimenticia fueron similares $(P>0.05)$ entre tratamientos. Sin embargo; para el peso del huevo, se puede observar que este fue mayor $(P<0.05)$ en las aves alimentadas con la dieta testigo (T1) y la dieta reducida con enzimas + probiótico (T3).

Cuadro 2: Variables productivas de gallinas Bovans de 42-54 semanas de edad alimentadas con dietas sorgo-soya-canola

\begin{tabular}{lllll}
\hline Dietas & $\begin{array}{l}\text { Postura } \\
(\%)\end{array}$ & $\begin{array}{l}\text { Peso } \\
\text { huevo }(\mathbf{g})\end{array}$ & $\begin{array}{l}\text { Consumo/ave/ } \\
\text { día }(\mathbf{g})\end{array}$ & $\begin{array}{l}\text { Conversión } \\
\text { alimenticia } \\
(\mathbf{g} / \mathbf{g})\end{array}$ \\
\hline 1) Dieta testigo & $92.2 \pm 1.9$ & $60.0 \pm 0.4 \mathrm{a}$ & $104 \pm 0.5$ & $1.88 \pm 0.04$ \\
2) Dieta reducida + enzimas & $92.8 \pm 1.7$ & $58.9 \pm 0.7 \mathrm{~b}$ & $104 \pm 0.8$ & $1.90 \pm 0.03$ \\
3) Dieta 2 + probiótico & $91.5 \pm 1.7$ & $59.2 \pm 0.4 \mathrm{ab}$ & $104 \pm 0.8$ & $1.91 \pm 0.03$ \\
\hline
\end{tabular}

Valores \pm error estándar.

a,b Valores con distinta literales son diferentes $(P<0.05)$.

En el Cuadro 3, se muestran datos obtenidos durante el experimento en la calidad del huevo. Los resultados de las Unidades Haugh, grosor del cascarón y color de la yema de huevo en base al abanico DSM, resultaron similares $(P>0.05)$ entre tratamientos.

Cuadro 3: Variables en la calidad de huevo de las gallinas de postura de 42 semanas de edad, alimentadas con dietas sorgo-pasta de soya-pasta de canola

\begin{tabular}{llll}
\hline Dietas & $\begin{array}{l}\text { Grosor cascarón } \\
(\boldsymbol{\mu m})\end{array}$ & $\begin{array}{l}\text { Unidades } \\
\text { Haugh }\end{array}$ & $\begin{array}{l}\text { Color de la yema } \\
\text { (abanico DSM) }\end{array}$ \\
\hline 1) Dieta testigo & $337 \pm 7.7$ & $90.8 \pm 2.8$ & $9.4 \pm 2.8$ \\
2) Dieta reducida + Enzimas & $352 \pm 7.2$ & $92.2 \pm 2.5$ & $9.2 \pm 0.4$ \\
3) Dieta 2 + Probiótico & $347 \pm 9.2$ & $91.2 \pm 1.8$ & $9.4 \pm 0.5$ \\
\hline
\end{tabular}

Valores \pm error estándar.

$(P>0.05)$.

En el Cuadro 4, se muestran los resultados promedio de los análisis de colesterol sérico, LDL, HDL y producción de IgA secretora en intestino. Se puede apreciar que no existió diferencia estadística $(P>0.05)$ entre los tratamientos. 
Cuadro 4: Cambios ocurridos en el contenido de colesterol sérico, LDH, HDL y IgA secretora en gallinas Bovans con dietas sorgo-pasta de soya-pasta de canola de 42-54 semanas de edad

\begin{tabular}{lllll}
\hline Dietas & $\begin{array}{l}\text { Colesterol } \\
(\mathbf{m g} / \mathbf{d l})\end{array}$ & $\begin{array}{l}\text { LDL } \\
(\mathbf{m g} / \mathbf{d l})\end{array}$ & $\begin{array}{l}\text { HDL } \\
(\mathbf{m g} / \mathbf{d l})\end{array}$ & $\begin{array}{l}\text { IgA secretora } \\
(\mathbf{\%})\end{array}$ \\
\hline 1) Dieta testigo & $100.2 \pm 26.2$ & $13.9 \pm 10.8$ & $30.0 \pm 2.5$ & $40.9 \pm 30.4$ \\
2) Dieta reducida+ enzimas & $109.7 \pm 15.7$ & $17.3 \pm 2.3$ & $37.0 \pm 5.2$ & $68.7 \pm 34.0$ \\
3) Dieta 2 + probiótico & $122.9 \pm 21.1$ & $16.2 \pm 0.9$ & $35.7 \pm 5.0$ & $63.0 \pm 27.9$ \\
\hline \multicolumn{4}{c}{ Valores \pm error estándar. } \\
$(P>0.05)$.
\end{tabular}

\section{Discusión}

Los resultados observados en el presente trabajo, coinciden con los de Wena et al ${ }^{(22)}$ quienes evaluaron un cóctel enzimático en dietas maíz-soya con menor contenido de nutrientes, y encontraron que se mejoró el valor alimenticio del alimento; por esta razón son utilizados en la industria de los alimentos para reducir los costos de formulación sin afectar el comportamiento productivo ${ }^{(8)}$; como lo realizado en este estudio, en donde las dietas reducidas en nutrientes bajo las recomendaciones nutricionales de la estirpe Bovans White, tuvieron menor contenido de pasta de soya y aceite.

Sobczak y Kozlowski ${ }^{(23)}$ evaluaron el efecto de adicionar Bacillus subtilis sobre la producción de huevo sin cambios significativos en el peso del huevo, porcentaje de postura, consumo de alimento y conversión alimenticia. Otros estudios también demuestran que el empleo de probióticos en dietas para gallinas, no tuvieron ninguna influencia sobre el rendimiento productivo ${ }^{(24)}$.

Se observó mayor peso del huevo en las dietas sin reducción de nutrientes, lo que sugiere probablemente que la reducción de $50 \mathrm{kcal}$ de EM en dietas sorgo-soya-canola+enzimas, no aportó la suficiente cantidad de EM por el complejo multienzimático. Sin embargo, la adición de enzimas y el probiótico incrementó el peso del huevo; probablemente debido a que los Bacillus subtilis del producto empleado, promovieron un microbioma más favorable y una mejor salud intestinal. Lo anterior estaría en parte de acuerdo a lo informado por Amerah et $a l^{(9)}$ que demostraron que las enzimas amilasas, xilanasas y proteasas solas o en combinación, incrementaron la digestibilidad de los polisacáridos no amiláceos contenidos en los ingredientes. No así, en otro trabajo ${ }^{(16)}$ donde estudiaron en pollos de engorda y emplearon fitasas solas o en combinación con xilanasas, amilasas, proteasas y un probiótico a base de Bacillus amyloliqueciens, sin efecto positivo en el rendimiento productivo. Sin embargo, la combinación incrementó la digestibilidad ileal 
aparente de azúcares y grasa con aumento en EM, además de reducir las poblaciones bacterianas patógenas.

Se ha estudiado ${ }^{(25)}$, la inclusión de carbohidrasas y proteasas en dietas maíz-soya-pasta de canola, las cuales incrementaron la ganancia de peso y la conversión alimenticia, además de mejorar la digestibilidad de proteína y la energía metabolizable; los resultados no coinciden con los obtenidos en el presente estudio, ya que no se mejoraron dichos parámetros productivos. En cuanto a los datos obtenidos en concentración de colesterol, HDL y LDH, no mostraron diferencia en este estudio; sin embargo, Salma et $a l^{(26)}$ emplearon un probiótico a diferentes concentraciones a base de Rhodobacter capsulatus en dietas maízsoya para gallinas, y reportaron que las dietas con la mayor concentración del probiótico, presentaron un aumento en las lipoproteínas de alta densidad HDL, colesterol e índice aterogénico en el suero. Recientemente, se ha reportado ${ }^{(3)}$ que la inclusión de 0, 250, 450 y 900 U/kg de xilanasas derivadas de la fermentación de Bacillus subtilis en dietas maíztrigo-pasta de soya para gallinas Hy-Line Brown, no mejoraron los parámetros productivos, sin embargo hubo efecto en el grosor del cascarón y las unidades Haugh; información que no concuerda con la del presente estudio.

\section{Conclusiones e implicaciones}

El empleo del complejo multienzimático compuesto de amilasas, proteasas y xilanasas y el probiótico a base de esporas de Bacillus subtilis incluido en dietas sorgo-soya-canola con las recomendaciones nutricionales de la estirpe Bovans White, permite reducir la EM en 50 $\mathrm{Kcal} / \mathrm{kg}$ y la proteína y aminoácidos esenciales lisina y metionina en $2 \%$, sin efecto perjudicial en el rendimiento productivo en gallinas Bovans White de 42 a 54 semanas de edad. No existieron cambios en los valores de IgA secretora intestinal ni de colesterol, HDL y LDH en suero al suplementar el complejo enzimático y el probiótico a base de Bacillus subtilis.

\section{Literatura citada:}

1. Hahn-Didde D, Purdum ESh. The effects of an enzyme complex in moderate and low nutrient-dense diets with dried distillers' grains with solubles in laying hens. J Appl Poult Res 2014;23(1):23-33.

2. Olukosi OA, Beeson LA, Englyst K, Romero LF. Effects of exogenous protease without or with carbohydrates on nutrient digestibility and disappearance of nonpolysaccharides in broiler chickens. Poult Sci 2015;94(11):2662-2669. 
3. Lei XJ, Lee KY, Kim IH. Performance, egg quality, nutrient digestibility, and excreta microbiota shedding in laying hens fed corn-soybean meal-wheat-based diets supplemented with xylanase. Poult Sci 2018;1(1):1-7.

4. Slominski BA. Recent advances in research on enzymes for poultry diets. Poult Sci 2011;90(10):2013-2023.

5. Bobeck EA, Nachtrieb NA, Batal $A B \dagger$, Persia ME. Effects of xylanase supplementation of corn-soybean meal-dried distiller's grain diets on performance, metabolizable energy, and body composition when fed to first-cycle laying hens. J Appl Poult Res 2014;23(1):174-180.

6. Gadde U, Kim WH, Oh ST, Lillehoj HS. Alternatives to antibiotics for maximinizing growth performance and feed efficiency in poultry: A review. Anim Health Res Rev 2017;18(1):26-45.

7. Liu W, Kim I. Effects of dietary xylanase supplementation on performance and functional digestive parameters in broilers fed wheat-based diets. Poult Sci 2016;96(3):566-573.

8. Bedford MR, Partridge GG. Enzymes in farm animal nutrition, 2nd ed. London, UK: CAB International; 2011.

9. Amerah AM, Romero LF, Awati A, Ravindran V. Effect of exogenous xylanase, amylase, and protease as single or combined activities on nutrient digestibility and growth performance of broilers fed corn-soy diets. Poult Sci 2017;96(4):807-816.

10. Cowieson AJ, Ravindran V. Effect of exogenous enzymes in maize-based diets varying in nutrient density for young broilers: Growth performance and digestibility of energy, minerals and amino acids. Br Poult Sci 2008;49(1):37-44.

11. Tang D, Hao S, Liu G, Nian F, Ru Y. Effects of maize source and complex enzymes on performance and nutrient utilization of broilers. Asian-Austral $\mathrm{J}$ Anim Sci 2014;27(6):1755-1762.

12. Romero LF, Sands JS, Indrakumar SE, Plumstead PW, Dalsgaard S, Ravindran V. Contribution of protein, starch, and fat to the apparent ileal digestible energy of cornand wheat-based diets in response to exogenous xylanase and amylase without or with protease. Poult Sci 2014;93(1):1-13.

13. Rinttilä T. Apajalahti J. Intestinal microbiota and metabolites Implications for broiler chicken health and performance. J Appl Poult Res 2013; 22(5):647-658. 
14. Latorre JD, Hernandez X, Kallapura G, Mencini A. Evaluation of germination, distribution, and persistence of Bacillus subtilis spores through the gastrointestinal tract of chickens. Poult Sci 2014;93(8):1793-1800.

15. Ribeiro LFT, Albino HS, Rostagno SLT, Barreto MI, Hannas D, Harrington FA, et al. Effects of the dietary supplementation of Bacillus subtilis levels on performance, egg quality and excreta moisture of layers. Anim Feed Sci Technol 2014;195(1):142-146.

16. Wealleans AL, Walsh MC, Romero LF, Ravindran V. Comparative effects of two multi-enzyme combinations and a Bacillus probiotic on growth performance, digestibility of energy and nutrients, disappearance of non-starch polysaccharides, and gut microflora in broiler chickens. Poult Sci 2017;96(4):4287-4297.

17. Romero LF, Parsons CM, Utterback PL, Plumstead PW, Ravindran V. Comparative effects of dietary carbohydrates without or with protease on the ileal digestibility of energy and amino acids and AME. Anim Feed Sci Technol 2017;181(1):35-44.

18. Li L, Xu CL, Ji C, Ma Q, Hao K, Jin ZY, Li K. Effects of a dried Bacillus subtilis culture on egg quality. Poult Sci 2006;85(2):364-368.

19. Sohail HK, Muhammad A, Nasir M. Effects of supplementation of multi-enzyme and multi-species probiotic on production performance, egg quality, cholesterol level and immune system in laying hens. J Appl Anim Res 2011;39(3):386-398.

20. Gómez VGG. Modulación nutricional de la inmunidad en pollo de engorda mediante el empleo de un estimulante (paredes de levaduras de Saccharomyces cerevisiae) [tesis doctorado]. México, CDMX: Universidad Nacional Autónoma de México; 2009.

21. IBM®SPSS®STATISTICS VER. 19.0.0@COPYRIGHT SPSS Inc. 1989 2010.:

22. Wena LC, Wanga YM, Zhoua Z, Jiangb Y, Wanga T. Effect of enzyme preparation on egg production, nutrient retention, digestive enzyme activities and pancreatic enzyme messenger RNA expression of late-phase laying hens. Anim Feed Sci Technol 2012;172(1):180-186.

23. Sobczak A, Kozlowski K. The effect of a probiotic preparation containing Bacillus subtilis ATCC PTA-6737 on egg production and physiological parameters of laying hens. Ann Anim Sci 2015;15(4):711-723.

24. Panda AK, Reddy MR, Rama Rao SV, Praharaj NK. Production performance, serum/yolk cholesterol and immune competence of White Leghorn layers as influenced by dietary supplementation with probiotic. Trop Anim Health Prod 2003;35(1):85-94. 
25. Toghyani M, Wu SB, Pérez-Maldonado RA, Iji PA, Swick RA. Performance, nutrient utilization, and energy partitioning in broiler chickens offered high canola meal diets supplemented with multicomponent carbohydrate and mono-component protease. Poult Sci 2017;96(12):3960-3972.

26. Salma U, Miah AG, Tareq KM, Maki T, Tsujii H. Effect of dietary Rhodobacter capsulatus on egg-yolk cholesterol in laying hen performance. Poult Sci 2007;86(7):714-719. 